\title{
Multiwavelength observations of the blazar BL Lacertae: a new fast TeV $\gamma$-ray flare
}

Q. Feng ${ }^{* a}$ for the VERITAS Collaboration; S. G. Jorstad ${ }^{b, c}$, A. P. Marscher ${ }^{b}$, M. L. Lister ${ }^{d}$, Y. Y. Kovalev ${ }^{e, f}$, A. B. Pushkarev ${ }^{g, e}$, T. Savolainen ${ }^{h, i, f}$, I. Agudo ${ }^{i}$, S. N. Molina ${ }^{j}$, J. L. Gomez ${ }^{j}$, V. M. Larionov ${ }^{c}$, G. A. Borman ${ }^{g}$, A. A. Mokrushina ${ }^{c}$, and P. S. Smith ${ }^{k}$

a Physics Department, McGill University, Montreal, QC H3A 2T8, Canada

E-mail: qi.feng2@mcgill.ca

${ }^{b}$ Institute for Astrophysical Research, Boston University, 725 Commonwealth Avenue, Boston, MA 02215, USA

${ }^{c}$ Astronomical Institute, St.Petersburg State University, Universitetskij Pr. 28, Petrodvorets, 198504 St.Petersburg, Russia

${ }^{d}$ Purdue University, 525 Northwestern Avenue, West Lafayette, IN 47907, USA

${ }^{e}$ Astro Space Center of Lebedev Physical Institute, Profsoyuznaya 84/32, 117997 Moscow, Russia

${ }^{f}$ Max-Planck-Institut für Radioastronomie, Auf dem Hügel 69, 53121 Bonn, Germany

${ }^{g}$ Crimean Astrophysical Observatory, 98409 Nauchny, Crimea, Russia

${ }^{h}$ Aalto University Metsähovi Radio Observatory, Metsähovintie 114, FI-02540 Kylmälä, Finland

${ }^{i}$ Aalto University Department of Electronics and Nanoengineering, PL 15500, FI-00076 Aalto,

Finland

${ }^{j}$ Instituto de Astrofísica de Andalucía (CSIC), Apartado 3004, E-18080 Granada, Spain

${ }^{k}$ Steward Observatory, University of Arizona, Tucson, AZ 85716, USA

\begin{abstract}
Observations of fast $\mathrm{TeV} \gamma$-ray flares from blazars reveal the extreme compactness of emitting regions in blazar jets. Combined with very-long-baseline radio interferometry measurements, they probe the structure and emission mechanism of the jet. We report on a fast $\mathrm{TeV} \gamma$-ray flare from BL Lacertae observed by VERITAS, with a rise time of about 2.3 hours and a decay time of about 36 minutes. The peak flux at $>200 \mathrm{GeV}$ measured with the 4-minute binned light curve is $(4.2 \pm 0.6) \times 10^{-6}$ photons $\mathrm{m}^{-2} \mathrm{~s}^{-1}$, or $\sim 180 \%$ the Crab Nebula flux. Variability in GeV $\gamma$-ray, $\mathrm{X}$-ray, and optical flux, as well as in optical and radio polarization was observed around the time of the TeV $\gamma$-ray flare. A possible superluminal knot was identified in the VLBA observations at $43 \mathrm{GHz}$. The flare constrains the size of the emitting region, and is consistent with several theoretical models with stationary shocks.
\end{abstract}

35th International Cosmic Ray Conference - ICRC2017

12-20 July, 2017

Bexco, Busan, Korea

\footnotetext{
*Speaker.

${ }^{\dagger}$ veritas.sao.arizona.edu
} 


\section{Introduction}

Some TeV blazars exhibit fast $\gamma$-ray variability, the timescale of which can be as short as a few minutes at very high energies $\left(100 \mathrm{GeV} \lesssim E_{\gamma} \lesssim 100 \mathrm{TeV}\right.$; VHE). Such variability has been observed in several BL Lac objects, including the prototype BL Lacertae (BL Lac hereafter) [1], located at a redshift of $z=0.069$ (an angular scale of $\approx 1.3 \mathrm{pc} / \mathrm{mas}$ ).

Long-term monitoring of BL Lac indicates that the source is not detectable in the $\mathrm{TeV} \gamma$-ray band except during flaring episodes, the most recent of which exhibited a flux of $\sim 125 \%$ of the Crab Nebula flux (C. U.) above $200 \mathrm{GeV}$ with a fast variability timescale of $13 \pm 4$ minutes in 2011 [1].

BL Lac exhibits both stationary radio cores/knots and superluminal radio knots [e.g. 2, and references therein]. Possible associations between the variability of superluminal radio knots and $\gamma$-ray flares have been investigated for the source [3, 1].

On 2016 Oct 5, the Very Energetic Radiation Imaging Telescope Array System (VERITAS) observed a sub-hour TeV $\gamma$-ray flare from BL Lac. A series of observations with the Very Long Baseline Array (VLBA) were taken at $43 \mathrm{GHz}$ and $15.4 \mathrm{GHz}$ over the span of a few months, revealing that a possible knot structure emerged around the time of the $\mathrm{TeV} \gamma$-ray flare. In this work, we report on the results of the aforementioned and other MWL observations, and discuss their implications.

\section{Observations, Data Analysis, and Results}

\subsection{VERITAS}

VERITAS is an array of four imaging atmospheric Cherenkov telescopes located in southern Arizona [see e.g. 4, for details]. BL Lac was observed at an elevated TeV $\gamma$-ray flux by VERITAS on 2016 Oct 5, with an exposure of 153.5 minutes after data quality selection. The data were analyzed using two independent analysis packages and pre-determined cuts optimized for lowerenergy showers. A detection with a statistical significance of $70.7 \sigma$ was made from the data of that night, with a time-averaged integral flux above $200 \mathrm{GeV}$ of $(2.24 \pm 0.06) \times 10^{-6}$ photons $\mathrm{m}^{-2} \mathrm{~s}^{-1}$.

Figure 1 shows the VERITAS light curves of BL Lac on 2016 Oct 5 with 4-minute and 30-minute bins. The measured peak flux of the 30-minute binned light curve is $(3.0 \pm 0.2) \times$ $10^{-6}$ photons $\mathrm{m}^{-2} \mathrm{~s}^{-1}$, corresponding to $\sim 125 \% \mathrm{C}$. U., and that of the 4 -minute binned light curve is $(4.2 \pm 0.6) \times 10^{-6}$ photons $\mathrm{m}^{-2} \mathrm{~s}^{-1}$, or $\sim 180 \% \mathrm{C}$. $\mathrm{U}$.

To quantify the rise and decay times of the TeV flare, we fitted the VHE $\gamma$-ray light curve with a piece-wise exponential function $F(t)=F_{0} e^{-\left|t-t_{\text {peak }}\right| / t_{\text {var }}}$, where $F_{0}$ is the peak flux, $t_{\text {peak }}$ is the peak time, and $t_{\mathrm{var}}$ is the rise or decay time.

The best parameters and their 99\% confidence intervals were determined using Markov chain Monte Carlo (MCMC) simulations, and are shown in Figure 1. The rise and decay timescales of the flare are determined to be $140_{-11}^{+25}$ minutes and $36_{-7}^{+8}$ minutes, respectively.

Further VERITAS observations of BL Lac were taken on Oct 6 and from Oct 22 to Nov 19 with 37.6-minute and 294.6-minute live exposure after data quality selection, respectively, neither of which led to a detection of the source. The integral flux upper limits $>200 \mathrm{GeV}$ at $99 \%$ confidence level on Oct 6 and between Oct 22 and Nov 19 were $2.0 \times 10^{-7}$ and $2.8 \times 10^{-8}$ photons $\mathrm{m}^{-2} \mathrm{~s}^{-1}$, respectively, assuming a power-law spectrum with a photon index of 3.3. 


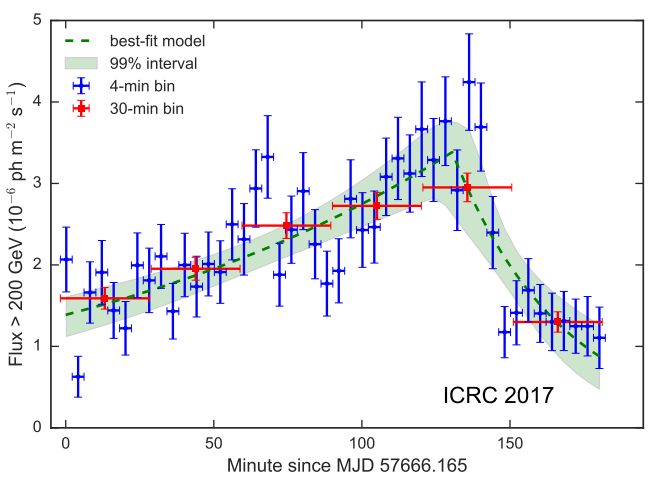

Figure 1: The VERITAS TeV $\gamma$-ray light curves of $\mathrm{BL} \mathrm{Lac}>200 \mathrm{GeV}$ on 2016 Oct 5 . The blue dots show the light curve in 4-minute bins, and the red squares show the light curve in 30-minute bins. The green dashed line and shaded region show the bestfit model and its 99\% confidence interval, respectively, using Markov chain Monte Carlo sampling.

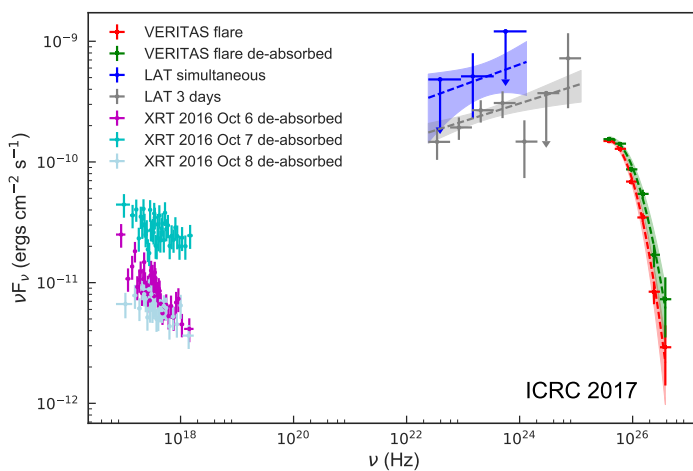

Figure 2: The $\gamma$-ray and X-ray SEDs measured by Fermi-LAT, VERITAS, and Swift-XRT. The FermiLAT SEDs, strictly simultaneous with VERITAS observations on 2016 Oct 5 and from the three days around it, are shown in blue and grey, respectively. The shaded regions represent $1-\sigma$ confidence intervals derived from the best-fit spectral model.

Motivated by the existence of multiple radio emission zones identified in VLBA data (see Subsection 2.5), we also fitted the light curve with a model including a constant flux baseline. In a multi-zone model, it is possible to have a larger emitting zone that varies slowly and can be adequately described by a constant baseline on the timescale considered, and a smaller, more energetic zone that is responsible for the fast flare described by the exponential components. With the more complex model, the best decay time is only $2.6_{-0.8}^{+6.7}$ minutes, with a baseline flux of $1.2_{-0.2}^{+0.1} \times 10^{-6} \mathrm{ph} \mathrm{m}^{-2} \mathrm{~s}^{-1}$. The baseline flux is higher than the upper limit obtained from the observations taken the following day, indicating the potential slower component varies on the timescale of $\sim 1$ day, consistent with the $\mathrm{GeV}$ observations. We note that with our limited statistics it is not possible to unambiguously reject either model.

A power-law fit to the VERITAS spectrum of BL Lac yields a $\chi_{\text {dof }}^{2}$ value of 34 and a bestfit photon index of $3.28 \pm 0.04$, insufficient to describe the data. A log parabola model with a fixed pivot energy of $0.2 \mathrm{TeV}$ fits the VERITAS spectrum better: $\frac{d N}{d E}=(2.22 \pm 0.07) \times 10^{-5} \times$ $\left(\frac{E}{0.2 \mathrm{TeV}}\right)^{\left[-(2.4 \pm 0.1)-(1.8 \pm 0.3) \log _{10}\left(\frac{E}{0.2 \mathrm{TeV}}\right)\right]} \mathrm{m}^{-2} \mathrm{~s}^{-1} \mathrm{TeV}^{-1}$, with a $\chi_{\text {dof }}^{2}$ value of 1.6. Both the observed and the de-absorbed $\mathrm{TeV} \gamma$-ray spectra are shown in Figure 2 in the $v F v$ representation.

\subsection{Fermi-LAT}

The Large Area Telescope (LAT) onboard the Fermi satellite is a pair-conversion $\gamma$-ray telescope sensitive to energies from $\sim 20 \mathrm{MeV}$ to $>300 \mathrm{GeV}$ [5]. An unbinned likelihood analysis was performed with the LAT ScienceTools v10r0p5 and Pass-8 P 8R2_SOURCE_V6_v0 6 instrument response functions, between $100 \mathrm{MeV}$ and $300 \mathrm{GeV}$ within $10^{\circ}$ from the position of BL Lac. As shown in the light curve in the first panel of Figure 3, BL Lac was in an elevated GeV $\gamma$-ray state when the $\mathrm{TeV}$ flare occurred. Its $\mathrm{GeV}$ flux varied by a factor of $\sim 2$ on a $\sim 1$ day timescale.

The GeV $\gamma$-ray SEDs strictly simultaneous with the TeV flare, as well as over a three-day interval around the time of the flare, are shown in Figure 2, with the best-fit power-law indices of $1.83 \pm 0.21$ and $1.85 \pm 0.07$, respectively. Both the $\mathrm{GeV}$ and $\mathrm{TeV} \gamma$-ray spectral indices obtained for this 2016 flare are comparable to those estimated for the 2011 flare. 


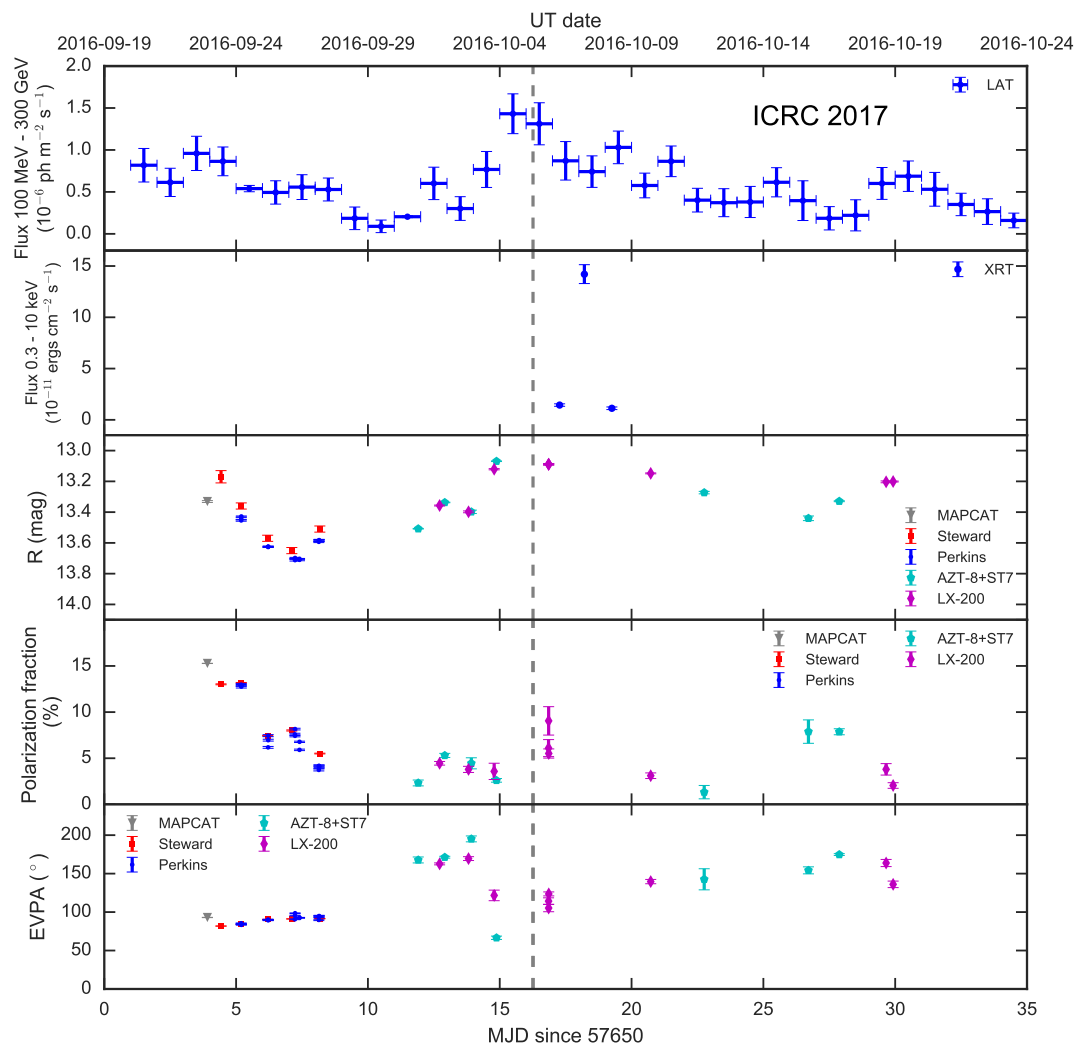

Figure 3: The one-month MWL light curves of BL Lac around the time of the VHE flare. The top panel shows the daily-binned GeV $\gamma$-ray light curve measured by Fermi-LAT. The second panel shows the dailybinned X-ray light curve measured by Swift-XRT. The lower three panels show the R-band photometric and polarimetric measurements taken by four instruments. The grey dashed line shows the peak time of the TeV flare observed by VERITAS.

\subsection{Swift XRT}

The X-Ray Telescope (XRT) onboard the Swift satellite is a grazing-incidence focusing X-ray telescope sensitive to photons in the $0.2-10 \mathrm{keV}$ energy range [6]. Swift followed up on BL Lac on 2016 Oct 6, 7, and 8, and no other observations were made in the one-month period around the time of the VHE flare. The XRT data, taken in the photon counting (PC) mode, were analyzed using the HEAs oft package (v6.19). Pile-up correction was necessary for data taken on 2016 Oct 7 and 8, and annular source regions with inner radii of 4 and 2 pixels, and outer radius of 20 pixels were used. The observations on Oct 7 consisted of two intervals of duration 486 seconds and 1422 seconds, the latter of which was discarded as a sustained dark stripe near the position of BL Lac contaminates the XRT image.

The X-ray spectrum was fitted with an absorbed power law model (po*wabs), and then deabsorbed with the best-fit neutral hydrogen column density values. The de-absorbed X-ray SEDs of BL Lac on 2016 Oct 6, 7, and 8 are shown in Figure 2. The X-ray emission from the source was the strongest and hardest on 2016 Oct 7 (two days after the TeV $\gamma$-ray flare) compared to the day before and after. The energy flux values on the three nights were $(1.4 \pm 0.1),(14.2 \pm 0.9)$, and $(1.1 \pm 0.1) \times 10^{-11} \mathrm{ergs} \mathrm{cm}^{-2} \mathrm{~s}^{-1}$, as shown in the second panel of Figure 3 .

\subsection{Optical facilities}

BL Lac was monitored in $\mathrm{R}$ band at a high cadence by a number of optical facilities, including 


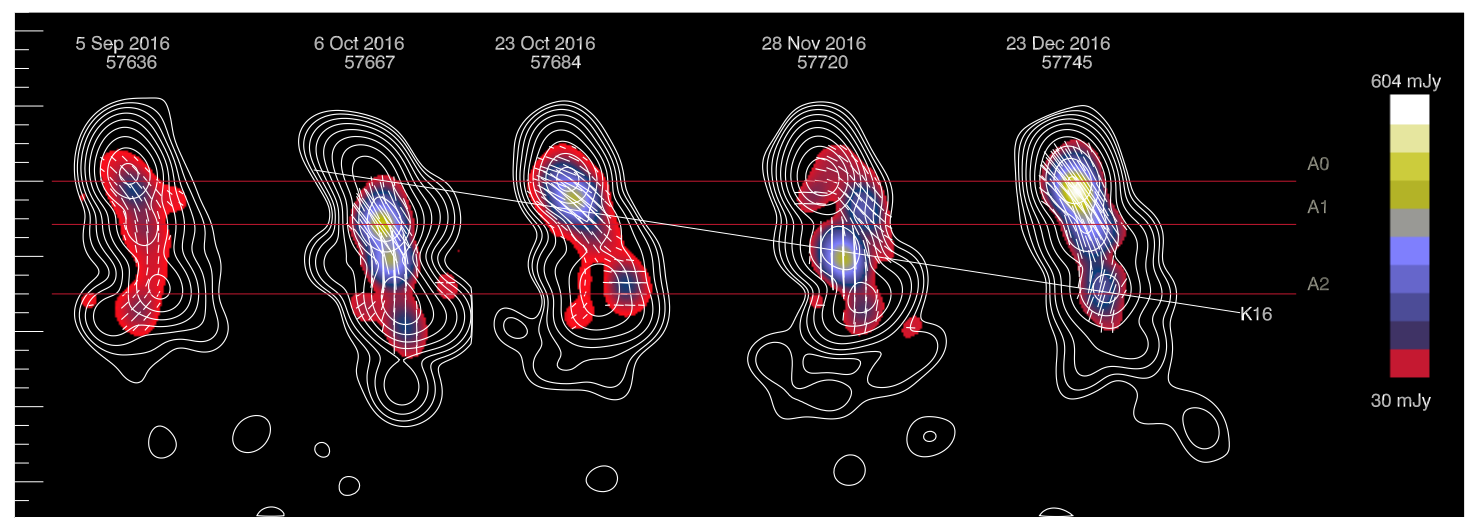

Figure 4: Total (contours) and linearly polarized (color code) intensity images of BL Lac from VLBA observations at $43 \mathrm{GHz}$. The images are convolved with a circular Gaussian restoring beam of FWHM= 0.1 mas. Tick marks are separated by 0.05 mas. The contours increase in multiples of 2 starting from $0.5 \%$ of the peak of $1.15 \mathrm{Jy} / \mathrm{beam}$. Red horizontal lines indicate the mean locations of the three quasi-stationary components, while the white line shows the motion of moving knot $K 16$.

the Steward Observatory[7] ${ }^{1}$, the AZT- 8 reflector of the Crimean Astrophysical Observatory, the Perkins telescope ${ }^{2}$, the LX-200 telescope in St. Petersburg, Russia, and the Calar Alto Telescope under the MAPCAT ${ }^{3}$ program.

The R-band flux and polarization measurements around the time of the VHE $\gamma$-ray flare are shown in Figure 3. The lower three panels show the R-band magnitude, polarization fraction, and electric vector position angle (EVPA), respectively. A $180^{\circ}$ shift is added to all the EVPA measurements after MJD 57662, so that the difference between adjacent EVPA measurements is minimized. An increase in the R-band flux, accompanied by a decrease of the optical polarization fraction and a deviation in the EVPA by $\sim 90^{\circ}$ were observed from the source a few days before the VHE flare. Such behaviour is consistent with the emergence of a radio knot observed by VLBA described below.

\subsection{VLBA}

BL Lac was observed throughout the period of interest at $43 \mathrm{GHz}$ with the VLBA under the VLBA-BU-BLAZAR monitoring program [8] and at $15.4 \mathrm{GHz}$ under the Monitoring Of Jets in Active galactic nuclei with VLBA Experiments (MOJAVE) program [9], the data calibration and imaging procedures of which were identical to those described by [10] and [9], respectively.

Figure 4 presents images of the parsec-scale jet of BL Lac at five epochs from 2016 September 5 to December 23. The linear resolution at the redshift of BL Lac is $0.13 \mathrm{pc}$ (corresponding to 0.1 mas) in projection on the sky and $1.8_{-0.4}^{+0.8} \mathrm{pc}$ for a viewing angle of $4.2^{\circ} \pm 1.3^{\circ}$ between the jet axis and line of sight [11].

As was the case in earlier observations [10,1, 12], the main structure of the compact jet consists of three quasi-stationary brightness peaks, designated as (from north to south) $A 0$ (used as the positional reference point), $A 10.12$ mas to the south, and $A 20.30$ mas to the south. The locations of $A 1$ and $A 2$ appear to fluctuate as moving emission features with superluminal apparent veloc-

\footnotetext{
${ }^{1}$ http://james.as.arizona.edu/ psmith/Fermi

${ }^{2}$ http://lowell.edu/research/research-facilities/1-8-meter-perkins/

$3_{\text {www. iaa.es/ iagudo/research/MAPCAT/MAPCAT.html }}$
} 

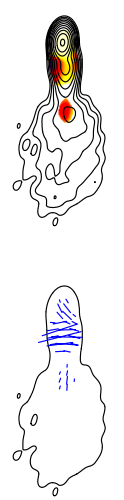

$2016 \operatorname{Sep} 26$
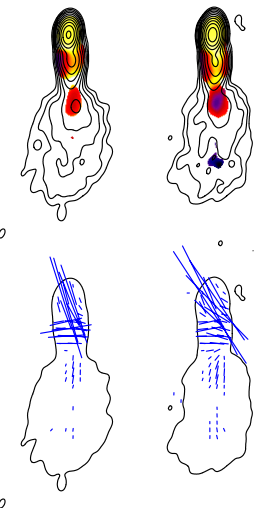

2016 oct 29
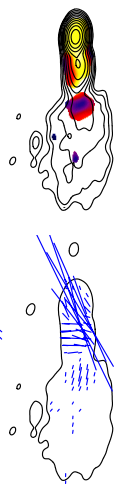

2016 Nov 06
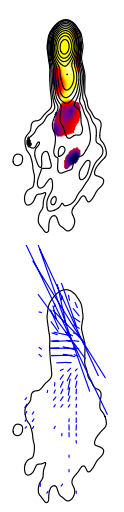

2016 Nov 12
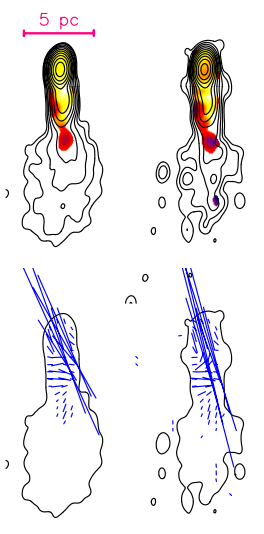

2016 Dec 10
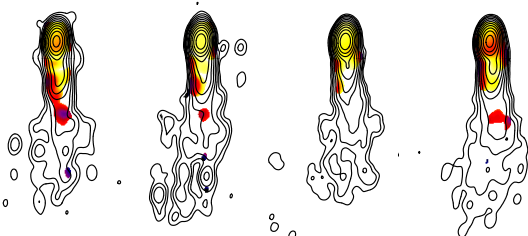

2016 Dec 26 :

Figure 5: Images of BL Lac from VLBA observations at $15.4 \mathrm{GHz}$ at nine epochs in 2016. A Gaussian restoring beam with dimensions $0.883 \times 0.56$ mas and a position angle -8.2 degrees was used. The contours show the total intensity, with a base contour of $1.1 \mathrm{mJy} / \mathrm{beam}$ and successive contours in the top row incrementing by factors of two. The colors in the top rows show the fractional polarization, and the blue line segments in the bottom rows show the EVPA. The length of the EVPA line segments corresponds to polarized intensity, the lowest of which shown is $0.5 \mathrm{mJy} / \mathrm{beam}$. The typical total and polarized intensity image rms are $0.09 \mathrm{mJy} / \mathrm{beam}$ and $0.1 \mathrm{mJy} /$ beam, respectively.

ities pass through the region. This combination of moving and stationary emission components complicates interpretation of the changing total and polarized intensity structure. Therefore, the interpretation that we offer to explain the variations seen the images is not unique.

A knot of emission with enhanced polarization, designated as $K 16$, appears to propagate down the jet. It moves by 0.23 mas between October 23 , when its centroid is $\sim 0.05$ mas south of $A 0$, and December 23, when it is 0.28 mas from $A 0$. This corresponds to an apparent speed of $6 c$, within the range typically observed in BL Lac $[1,3,10,11,12]$. Extrapolation back to October 6 places the knot 0.01 mas north of the centroid of $A 0$, within the $A 0$ emission region given its angular size of $0.03 \pm 0.02$ mas [11]. This interpretation implies that the VHE flare occurred as the knot crossed the "core," which has been interpreted as a standing shock located $\sim 1 \mathrm{pc}$ from the black hole [3].

The VLBA images at $15.4 \mathrm{GHz}$ reveal the evolution of jet structures further away from the central source on a larger spatial scale compared to the $43 \mathrm{GHz}$ images, as a result of a steep spectrum of optically thin synchrotron emission of outer jet regions. Figure 5 shows that the fractional polarization of the stationary core of BL Lac at $15.4 \mathrm{GHz}$ suddenly dropped on $2016 \mathrm{Dec} 26$, and gradually increased in the two epochs afterward. A small region with enhanced polarized intensity and distinct EVPA south of the core appeared on Dec 26, which may correspond to the knot $K 16$ observed at $43 \mathrm{GHz}$. However, we note that there is no indication of the same region in the total intensity map at $15.4 \mathrm{GHz}$.

\section{Discussion}

For the second time, a fast $\mathrm{TeV} \gamma$-ray flare from BL Lac was observed coincidentally with the appearance of a candidate superluminal radio knot. This suggests a possible association between the fast VHE $\gamma$-ray flare and the appearance of the superluminal radio knot for the source, similar to that reported by [1].

The fastest timescale of a flare (in this case the decay time) can be used to put an upper limit on the size of the emitting region, as $R \leq \mathrm{ct} t_{\text {decay }} \delta /(1+z)$, where $c$ is the speed of light, $z$ is the 
redshift of the source, and $\delta$ is the Doppler factor of the jet ${ }^{4}$. Taking the values of the mass of the central black hole $M_{\mathrm{BH}} \sim 3.8 \times 10^{8} M_{\odot}$ [13], the Doppler factor $\delta \sim 24$ [2], and the best-fit $t_{\text {decay }}=36_{-7}^{+8}$ minutes, the upper limit of the size of the emitting region is estimated as $R \lesssim 11.9 R_{S}$, where $R_{s}$ is the Schwarzschild radius.

The decay time of the observed VHE $\gamma$-ray flare was faster than the rise time. This is uncommon and may be caused by an abrupt stop of the high-energy particle injection [see e.g. 14]. The enhancement in the $\mathrm{GeV} \gamma$-ray and X-ray flux of BL Lac contemporaneous with the TeV flare provides evidence for strong activity of the relativistic particles in the jet, but not enough information for any abrupt stop.

In the model proposed by [15], the radio core is a structure containing a conical shock, perhaps with a Mach disk at its apex, downstream of the base of the jet. Turbulent cells of plasma first pass through the conical shock, where electrons are accelerated. The plasma then passes the Mach disk, where a fast $\gamma$-ray flare can happen via inverse-Compton scattering. After the Mach disk, a conical rarefaction can cause the plasma flow to expand and accelerate and appear as a superluminal radio knot. Polarization changes including a drop in the polarization fraction (due to the new magnetic field of the passing plasma cancelling that of the stationary core) and a swing in the polarization angle (as the passing plasma becomes brighter and dominant over the stationary core) are predicted in a similar model [16]. This is consistent with the optical polarization measurements from shortly before the VHE $\gamma$-ray flare, as well as the VLBA images at 43 and $15.4 \mathrm{GHz}$ afterward.

An alternative explanation of both the VHE $\gamma$-ray flare and the superluminal radio knot of BL Lac is the breakout of a recollimation shock zone [2]. In this model, a stationary knot can be carried away by the relativistic underlying flow and become a superluminal knot. During this process, a magnetic reconnection event can occur, leading to the observed fast flare. In the case of the 2016 flare of BL Lac, there is no evidence for the disruption or breakout of a stationary knot. Although it is possible that the recollimation zone reformed quickly between VLBA epochs and therefore wasn't sampled by the observations.

\section{Acknowledgments}

VERITAS is supported by grants from the U.S. Department of Energy Office of Science, the U.S. National Science Foundation and the Smithsonian Institution, and by NSERC in Canada. We acknowledge the excellent work of the technical support staff at the Fred Lawrence Whipple Observatory and at the collaborating institutions in the construction and operation of the instrument. The VERITAS Collaboration is grateful to Trevor Weekes for his seminal contributions and leadership in the field of VHE $\gamma$-ray astrophysics, which made this study possible.

The research at Boston University was supported in part by NASA Fermi Guest Investigator Program grant NNX14AQ58G. The VLBA is an instrument of the Long Baseline Observatory (LBO). The LBO is a facility of the National Science Foundation operated under cooperative agreement by Associated Universities, Inc. The MOJAVE program is supported under NASA-Fermi grant NNX15AU76G. Data from the Steward Observatory spectropolarimetric monitoring project were used. This program is supported by Fermi Guest Investigator grants NNX15AU81G. IA acknowledges support by a Ramón y Cajal grant of the Ministerio de Economía y Competitividad (MINECO) of Spain. The MAPCAT program was funded in part by MINECO through grants

\footnotetext{
${ }^{4} \delta=[\Gamma(1-\beta \cos \theta)]^{-1}$, where $\Gamma$ is the bulk Lorenz factor of the jet and $\theta$ is the viewing angle.
} 
AYA2010-14844, AYA2013-40825-P, and AYA2016-80889-P, and by the Regional Government of Andalucía through grant P09-FQM-4784.

\section{References}

[1] T. Arlen et al. Rapid TeV Gamma-Ray Flaring of BL Lacertae. In: ApJ 762, 92 (Jan. 2013), p. 92. arXiv: 1211.3073 [astro-ph.HE].

[2] O. Hervet, C. Boisson, and H. Sol. An innovative blazar classification based on radio jet kinematics. In: A\&A 592, A22 (July 2016), A22. arXiv: 1605.02272 [astro-ph.HE] .

[3] A. P. Marscher et al. The inner jet of an active galactic nucleus as revealed by a radio-to- $\gamma$ ray outburst. In: Nature 452 (Apr. 2008), pp. 966-969.

[4] J. Holder. VERITAS: Status and Highlights. In: International Cosmic Ray Conference 12 (2011), p. 137. arXiv: 1111.1225 [astro-ph.HE] .

[5] W. B. Atwood et al. The Large Area Telescope on the Fermi Gamma-Ray Space Telescope Mission. In: ApJ 697 (June 2009), pp. 1071-1102. arXiv: 0902.1089 [astro-ph. IM] .

[6] D. N. Burrows et al. The Swift X-Ray Telescope. In: Space Sci. Rev. 120 (Oct. 2005), pp. 165195. eprint: astro-ph/0508071.

[7] P. S. Smith et al. Coordinated Fermi/Optical Monitoring of Blazars and the Great 2009 September Gamma-ray Flare of 3C 454.3. In: ArXiv e-prints (Dec. 2009). arXiv: 0912 . 3621 [astro-ph.HE].

[8] S. Jorstad and A. Marscher. The VLBA-BU-BLAZAR Multi-Wavelength Monitoring Program. In: Galaxies 4 (Oct. 2016), p. 47.

[9] M. L. Lister et al. MOJAVE: Monitoring of Jets in Active Galactic Nuclei with VLBA Experiments. V. Multi-Epoch VLBA Images. In: AJ 137 (Mar. 2009), pp. 3718-3729. arXiv: 0812.3947.

[10] S. G. Jorstad et al. Polarimetric Observations of 15 Active Galactic Nuclei at High Frequencies: Jet Kinematics from Bimonthly Monitoring with the Very Long Baseline Array. In: AJ 130 (Oct. 2005), pp. 1418-1465. eprint: arXiv: astro-ph/ 0502501.

[11] S. G. Jorstad, A. P. Marscher, and D. A. Morozova. In: ApS submitted (May 2017).

[12] A. E. Wehrle et al. Erratic Flaring of BL Lac in 2012-2013: Multiwavelength Observations. In: ApJ 816, 53 (Jan. 2016), p. 53.

[13] Z.-Z. Wu, M.-F. Gu, and D.-R. Jiang. The debeamed luminosity, sychrotron peak frequency and black hole mass of BL Lac objects. In: Research in Astronomy and Astrophysics 9 (Feb. 2009), pp. 168-178. arXiv: 0804.1180.

[14] K. Katarzyński, H. Sol, and A. Kus. The multifrequency variability of Mrk 421. In: A\&A 410 (Oct. 2003), pp. 101-115.

[15] A. P. Marscher. Turbulent, Extreme Multi-zone Model for Simulating Flux and Polarization Variability in Blazars. In: ApJ 780, 87 (Jan. 2014), p. 87. arXiv: 1311 . 7665 [astro-ph. HE] .

[16] H. Zhang, X. Chen, and M. Böttcher. Synchrotron Polarization in Blazars. In: ApJ 789, 66 (July 2014), p. 66. arXiv: 1401.7138 [astro-ph.HE] . 